\title{
Green, orange, red, and far-red optogenetic tools derived from cyanobacteriochromes
}

\author{
Jaewan Jang ${ }^{1}$, Sherin McDonald ${ }^{2}$, Maruti Uppalapati ${ }^{2 *}$, G. Andrew Woolley ${ }^{1 *}$ \\ ${ }^{1}$ Department of Chemistry, University of Toronto, 80 St. George St., Toronto, ON, M5S 3H6, \\ Canada \\ ${ }^{2}$ Department of Pathology and Laboratory Medicine, University of Saskatchewan, Saskatoon, \\ Saskatchewan S7N 5E5, Canada
}

* To whom correspondence should be addressed andrew.woolley@utoronto.ca; maruti.uppalapati@usask.ca 


\begin{abstract}
Existing optogenetic tools for controlling protein-protein interactions are available in a limited number of wavelengths thereby limiting opportunities for multiplexing. The cyanobacteriochrome (CBCR) family of photoreceptors responds to an extraordinary range of colors, but light-dependent binding partners for CBCR domains are not currently known. We used a phage-display based approach to develop small ( 50-residue) monomeric binders selective for the green absorbing state $(\mathrm{Pg})$, or for the red absorbing state $(\mathrm{Pr})$ of the CBCR Am1_c0023g2 with a phycocyanobilin chromophore and also for the far-red absorbing state (Pfr) of $A m 1 \_c 0023 g 2$ with a biliverdin chromophore. These bind in a 1:1 mole ratio with $\mathrm{K}_{\mathrm{DS}}$ for the target state from 0.2 to $2 \mu \mathrm{M}$ and selectivities from 10 to 500 -fold. We demonstrate green, orange, red, and far-red light-dependent control of protein-protein interactions in vitro and also in vivo where these multicolor optogenetic tools are used to control transcription in yeast.
\end{abstract}

Keywords: photo-control, GAF, optogenetics, CBCR, phage display 


\section{Introduction}

Optogenetic tools that enable the control of protein-protein interactions are highly desirable for numerous cell biology applications $(1,2)$. Most available tools of this kind are naturally occurring photoswitchable binding partners (e.g. Cry2/CIBN, PhyB/PIF3, BPhP1/PpsR2) (3-5). Protein engineering approaches have been employed to develop improved photoswitchable protein-protein interaction modules based primarily on LOV domains $(1,6,7)$. While powerful, current tools have numerous limitations. For example, some of them (e.g. Gigantea, Cry, Phy) (8) are large and/or oligomeric and thus may perturb the folding and function of the proteins they are fused to $(3,9)$. While there are numerous examples of lightinduced protein-protein association, light induced dissociation is rare (AsLOV/zDark and cPYP/BoPD $(10,11))$. Most significantly, however, the currently available range of colors is incomplete - the large phytochrome systems respond to red/far red-light (3) but the vast majority of optogenetic tools for controlling protein-protein interactions respond to blue light (www.optobase.org)(12). As a result, opportunities for multiplexing optogenetic tools, or for using optogenetic tools together with the wide range of available fluorescent reporters are limited (3).

The cyanobacteriochromes (CBCRs), a superfamily of photoreceptor proteins discovered about ten years ago in cyanobacteria $(13,14)$ respond to a wide range of colors. These proteins typically consist of multiple subdomains called GAF domains (cGMPphosphodiesterase/adenylate cyclase/FhlA) linked in tandem (15). A conserved cysteine in the GAF domain covalently binds a bilin chromophore (typically phycocyanobilin (PCB)) via a thioether bond to the $\mathrm{C} 3^{1}$ atom and irradiation triggers $\mathrm{E} / \mathrm{Z}$ isomerization across the $\mathrm{C} 15$ double bond of the chromophore (16). Changes in the GAF protein environment around the bilin 
chromophore tune the wavelength of absorption to an extraordinary degree $(14,16)$. Recent discoveries have identified members of the CBCR family that respond to virtually any wavelength in the visible range from the far-red to the UV $(14,17-21)$. In addition to their wide range of colors, GAF domains are small (<200 residues) and can function as monomers $(22,23)$ - both desirable features for optogenetic tools.

Photoisomerization of the bilin chromophore leads to conformational changes in the GAF domain (24). A recent structural study of the red/green cyanobacteriochrome, NpR6012g4, showed that upon isomerization, a small segment of the protein near the chromophore changes from an irregular to a helical structure and subtler structural rearrangements are propagated throughout the protein (25). It has been hypothesized that GAF conformational changes modulate the dimerization propensity of CBCRs and/or the conformation of pre-formed dimers and this, in turn, regulates the activity of C-terminal effector domains (23). Conformational changes propagated via the $\mathrm{C}$-terminal linker have also been proposed to directly alter the conformation of the attached effector domain without stable dimerization (26). No naturally occurring light-dependent binding partners for CBCRs have been reported.

Recently we developed a phage-display approach to finding binding partners for photoswitchable proteins for which no known binding partner exists (10). The binding partners were developed by randomizing a surface on the small stable 3-helix bundle GA domain using a customized codon set (10). Here we apply a slightly modified approach to discover binding partners for a GAF domain from the CBCR family. As a target we chose Am1_c0023g2, a red/green GAF domain from Acaryochloris marina, that can bind two distinct bilins - PCB or biliverdin (BV) (27). When bound to PCB, Am1_c0023g2 forms a thermostable red-absorbing (Pr) state. When irradiated with red light $(680 \mathrm{~nm})$, the Pr state converts to a green absorbing 
state $(\mathrm{Pg})$. In the dark the $\mathrm{Pg}$ state exhibits very slow thermal reversion $\left(\tau_{1 / 2}>24 \mathrm{~h}, 25^{\circ} \mathrm{C}\right)$ to the $\mathrm{Pr}$ state. Irradiation of the Pg state with green light $(525 \mathrm{~nm})$ rapidly and quantitatively converts the protein back to the Pr state. When $A m 1 \_c 0023 g 2$ binds the more extensively conjugated BV chromophore, it forms a thermostable far-red absorbing (Pfr) state. The Pfr state can be converted using $750 \mathrm{~nm}$ light to an orange absorbing (Po) state, which reverts thermally to the Pfr state $\left(\tau_{1 / 2}=180 \mathrm{~min}, 25^{\circ} \mathrm{C}\right)$ or can be rapidly switched back to the Pfr state with orange light $(595 \mathrm{~nm})$. Thus, by changing the nature of the bilin chromophore loaded onto the protein, Am1_c0023g2 can be made responsive to either green, orange, red or far-red light. Optogenetic tools that respond to green light are currently limited to complex cobalamin based systems (28, 29) and CcaS/CcaR or cPAC systems that have predefined functions - gene expression (30), or adenylyl cyclase activity respectively (26). In addition, small, monomeric optogenetic tools that operate with far-red light are useful since far-red absorption provides deep tissue penetration ability for use in living animals (31). Proteins that operate using BV are of particular interest for studies in living animals where BV, though not PCB, is available via heme metabolism $(27,32)$. 


\section{Materials and Methods}

Production of Am1_c0023g2 PCB and BV

For production of Am1_c0023g2 in vitro the gene was subcloned into a pET24b vector containing a C-terminal poly His (6x) tag and apo-protein was expressed in E. coli BL21(DE3). A two-fold molar excess of purified PCB or BV (Frontier Scientific) in DMSO was added as described in the SI. For in vivo chromophore reconstitution, the gene was subcloned into a pBAD-HisC vector with a C-terminal 6x His-tag. E. coli strain LMG194 containing pPL-PCB (a kind gift from J.C. Lagarias) or pWA23h (a kind gift from V.V. Verkhusha) was transformed with pBAD-Am1_c0023g2 and the protein was expressed and purified as described in the SI.

\section{Phage display-based screening}

A M13 phage pVIII library based on the GA domain described previously (10) was used to find binders for the Pg, Pr, Po or Pfr states of Am1_c0023g2 using the detailed protocol described in the SI. The GA domain phage library was first depleted for binders to the apo protein. A second negative selection was carried out against the off-target state, followed by a positive selection on target. The resulting library of positive clones obtained after 3 rounds of selection was subcloned into a pIII phagemid and 100 single clonal phage from each of the positive pools were screened for binding using phage-based ELISAs (33). For affinity maturation, biased libraries were constructed in the pIII display format, based on doped oligonucleotides specific for lead clones BAm-green1.0 and BAm-red1.0 as described in the SI.

In vitro characterization of binders:

Selected binders were subcloned from pIII phagemids into the pET24b expression vector containing a C-terminal poly His (6x) tag and expressed and purified from E. coli. Size 
exclusion binding assays were performed by mixing Am1_c0023g2 PCB or BV $(80 \mu \mathrm{M})$ with each binder $(80 \mu \mathrm{M})$ in PBS and injecting onto a Superdex 75 10/300 GL size exclusion column maintained in the dark or while irradiating with either red $(680 \mathrm{~nm})$, green $(525 \mathrm{~nm})$, or far red $(750 \mathrm{~nm})$ light. ITC experiments were performed using a MicroCal VP-ITC MicroCalorimeter. Titration experiments were performed at $25^{\circ} \mathrm{C}$. The syringe contained the binder, BAM-red1.0 or BAm-green1.3, at $550 \mu \mathrm{M}$ or $750 \mu \mathrm{M}$, respectively. The cell contained either Am1_c0023g2 PCB exo $(45 \mu \mathrm{M}), A m 1 \_c 0023 g 2$ PCB endo $(42 \mu \mathrm{M}), A m 1 \_c 0023 g 2$ BV exo $(45 \mu \mathrm{M})$ or Am1_c0023g2 BV endo $(42 \mu \mathrm{M})$. Thermogram data were integrated using NITPIC (34) and binding analysis was carried out using SEDPHAT and recommend protocols $(35,36)$.

Yeast two-hybrid assays

The strains Y2HGOLD and Y187 were purchased from Clontech. pGAL4AD-x and pGAL4BD-y plasmids were purchased from Addgene (28246 and 28244) as pGAL4AD-CIB1 and pGAL4BD-Cry2 (4). The Am1_c0023g2 gene was PCR amplified and inserted (Gibson Assembly) into either the pGAL4AD or the pGAL4BD vector by replacing a CIB1 or Cry2 gene, respectively. Binder constructs were also subcloned into both plasmids using the same protocol. Strains containing pGAL4AD-x and pGAL4BD-y, respectively, were mated and selected on synthetic media lacking leucine and tryptophan. A single colony was picked and used to inoculate media containing $10 \mu \mathrm{M}$ PCB or $40 \mu \mathrm{M}$ BV (Frontier Scientific). Cultures were grown under different illumination conditions (green, red, far-red, dark), cells were harvested and $\beta$ galactosidase activity was measured following the manufacturers protocol as described in the SI. The experiment was performed in quadruplicate. 


\section{Results and Discussion}

Am1_c0023g2 engineering, expression and reconstitution:

Cyanobacteriochromes occur naturally as multi-domain proteins that may dimerize to varying extents (23). Since use as an optogenetic tool for controlling protein-protein interactions is simpler if the interacting protein partners are monomers, we sought to prevent dimerization of Am1_c0023g2. Dimerization of cyanobacterial GAF domains is analogous to that of PHY-GAFPAS domains from bacterial phytochromes (24). Previous work successfully monomerized a bacterial phytochrome by preventing a coiled-coiled interaction involving the $\mathrm{C}$-terminal helix (32). We therefore mutated Leu159 on the C-terminal helix to Lys and also truncated the Nterminal helix of Am1_c0023g2 to prevent homodimer formation (see sequences in SI Fig. S1).

(a)

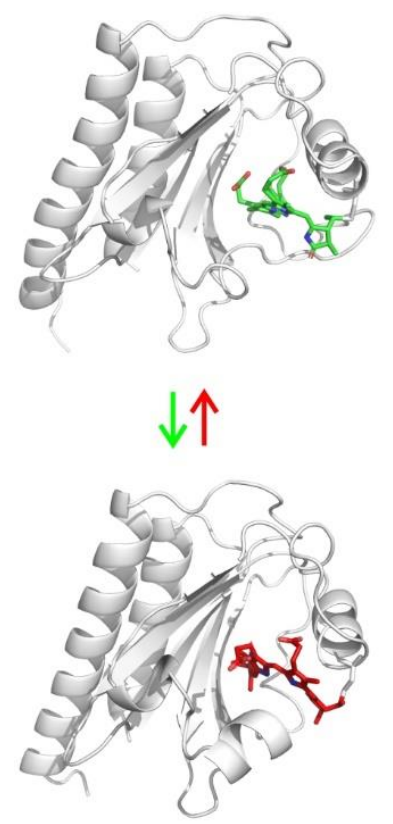

(b)

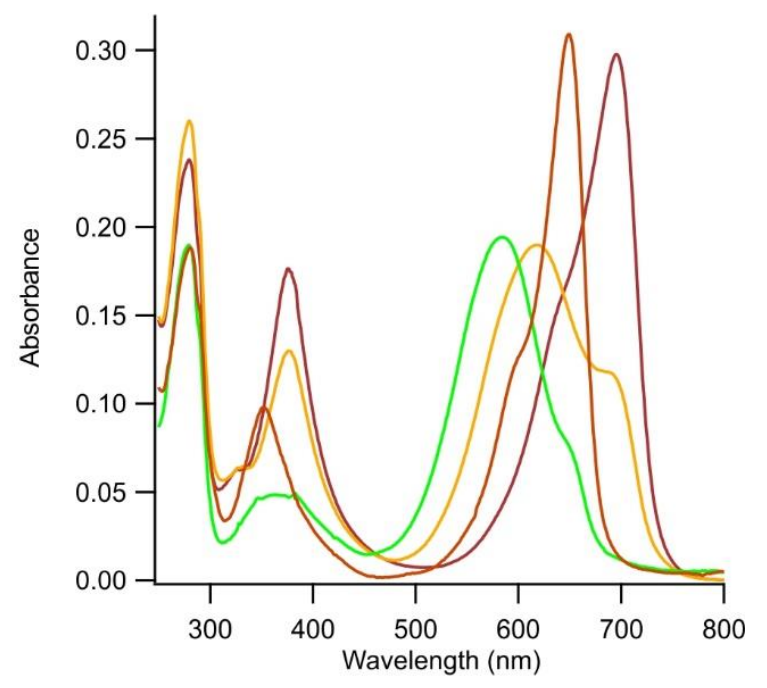

Figure 1. (a) Modeled structures of Pg and Pr states of Am1_c0023g2 PCB. Structures were generated by threading the Am1_c0023g2 sequence through models of NpR6012g4 (PDBIDs: 6BHN Pr state; 6BHO, Pg state (25)) using Phyre2 (37) (b) Absorbance spectra of Am1_c0023g2 with exogenous PCB incorporation in the Pr state (red line) and Pg state (green line) and with exogenous BV incorporation in the Pfr state (far-red line) and the Po state (orange line). 
We expressed this variant of Am1_c0023g2 with a C-terminal His-tag using an E. coli expression system, either with exogenous addition of PCB or BV or via coexpression of the enzymes to make each bilin (38). The protein eluted at a size corresponding to a monomeric, 20 kDa protein when analyzed using size exclusion chromatography (SEC) (Fig. S2). Spectra obtained matched those described by Narikawa and colleagues (27) except that the maximum wavelength of the Pg state observed via exogenous addition of PCB was red-shifted compared to that obtained for the Pg state of Am1_c0023g2 reconstituted via coexpression of heme oxygenase (HO1) and phycocyanobilin:ferredoxin oxidoreductase (PcyA) to produce PCB in vivo (Fig. 1, Fig. S3). Spectra for BV-loaded Am1_c0023g2 were insensitive to whether the bilin was added exogenously or endogenously (Fig. 1, Fig. S3). Differences in chromophore absorption spectra that depend on the mode of chromophore loading have been noted previously for other CBCRs $(39,40)$ and have been attributed to local conformational differences in the bilin binding pocket that may occur depending on whether chromophorylation occurs co-translationally or posttranslationally (39). Presumably the same sensitivity to local environment that leads to the extraordinary degree of color tuning seen with the CBCR photoreceptor family can also lead to spectral differences if conformational changes in the bilin binding pocket occur with protein expression changes (e.g. changes in host organism, rate of synthesis, temperature etc.). Additionally, a consistent observation for both exogenously-assembled and endogenouslyassembled preparations is that significant amounts of apoprotein are often present $(18,40)$. These features of the CBCR family are important to bear in mind when employing CBCRs as optogenetic tools in heterologous systems since the mode of chromophore loading (e.g. adding to cells in culture (41), or via coexpression of chromophore biosynthesis genes $(40,42)$ may lead to a mixture of apoprotein and distinct subsets of holoproteins in specific cases. Since we anticipate 
that initial applications of multicolour GAF-based optogenetic tools will be cells in culture where exogenous addition of chromophore is straightforward and provides a convenient way of commencing an experiment $(10,41)$ and because the exogenous reconstitution of Am1_c0023g2 with PCB and BV reliably produced $\sim 100 \%$ holo protein in our hands, we first focused on finding binding partners to the exogenously produced forms of the proteins. In addition, we aimed to find binding partners that would not recognize the apoprotein.

Phage-display based discovery of binding partners for Am1_c0023g2

In previous work we successfully used pVIII displayed libraries of the 3-helix bundle GA domain to find binders for both the blue-light and dark-adapted states of LOV and PYP photoreceptors (10). We used the same library to search for binders to distinct conformational states of apo- and holo- Am1_c0023g2 with either PCB or BV chromophores. First, we carried out a negative selection step to deplete the phage pool for binders to the apo-protein. To select for binders to the Pg state, a second negative selection step to remove binders for the Pr state was carried out by exposing the library to a plate containing holo-Am1_c0023g2 PCB in the Pr state. Unbound phage from this plate were then transferred to a third plate with holo-Am1_c0023g2 PCB in the Pg state. This plate was washed extensively, then binders were eluted and amplified. This three-step selection process was repeated two more times. Selection for binders to the Pr, Po, and Pfr states was carried out in an exactly analogous manner. The resulting phage pools were then subcloned into a pIII display phagemid as described previously (10). Since there are fewer copies of the GA domain displayed in the pIII fusion compared to the pVIII fusion context per phage this step may select for higher affinity binders by decreasing the likelihood of aviditymediated binding. In addition, clones that function in both pIII and pVIII contexts are expected 
to be less sensitive to the presence of surrounding phage proteins for their function. Perhaps

because of the subtle conformational change between photo-states that was being selected for,

very limited enrichment of phage libraries was observed at the pool level. We therefore screened

96 single clones using phage ELISA assays to search for photo-state selective binders.
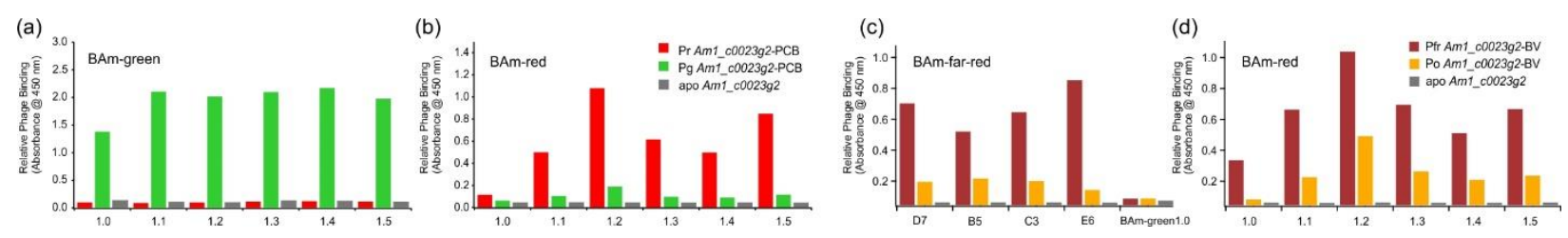

Figure 2 Phage-based ELISAs of BAm-green, BAm-red, and BAm-far-red sequences and their affinity matured variants. (a) BAm-green 1.0 was selected against the Pg state of Aml_c0023g2-PCB. BAm-green1.1-1.5 are affinity-matured variants. (b) BAm-red 1.0 was selected against the Pr state of Am1_c0023g2 PCB. BAm-red1.1-1.5 are affinity-matured variants. Although the signal from BAm-red1.0 is lower, the specificity is higher than for 1.11.5. (c) BAm-far-red clones were selected against the Pfr state of Am1_c0023g2 BV. No clones selective for the Po state of Am1_c0023g2 BV were found. BAm-green 1.0 does not bind to either state of Am1_c0023g2 BV. (d) BAmred and affinity-matured variants selectively bind the Pfr state of Am1_c0023g2 BV.

Clones were selected based on their apparent affinity and selectivity in phage-based ELISA assays (Fig. 2). The best clone for state selective binding to Am1_c0023g2 PCB in the Pg state, designated BAm-green1.0 (for binder of Am1_c0023g2-Pg) showed a 13-fold better binding to the Pg versus the Pr state. The best clone for selective binding to holo-Am1_c0023g2 PCB in the Pr state, designated BAm-red1.0 (for binder of Am1_c0023g2-Pr) showed 7-fold better binding for Pr over Pg. Both clones showed no detectable binding to the apo form of Am1_c0023g2 (Fig. 2). While no Po-state selective binders Am1_c0023g2 BV were found, several sequences were found to be selective for the Pfr state (Fig. 2c). Interestingly, the Pr-state binder BAm-red1.0 was also found to bind the Pfr state with similar affinity and selectivity to those binders directly selected for the Pfr state. In contrast, BAm-green 1.0 did not bind the Po state of GAF2-BV (Fig. 2c). We therefore opted to pursue BAm-red1.0 as both a Pr and a Pfr state binder. 
In the absence of structural information, it is not clear which of the previously randomized positions of the GA domain scaffold make contact with the target protein in each state. Therefore, we used a soft-randomization approach to improve the affinity of binders (43). We constructed biased pIII phage libraries based on BAm-green1.0 and BAm-red1.0 sequences. Here each of the nucleotides encoding the randomized positions was substituted with a doped mix such that the native nucleotide in the parent DNA sequence was doped at $70 \%$ while the rest of the 3 nucleotides occured at 10\% frequency each (see Methods). This allows for approximately $40 \%$ bias for the native amino acid in each randomized position, while allowing the occurrence of the other 19 amino acids at lower frequencies. Four rounds of selection were carried out using the same protocol as described for the naïve library, then $\sim 24$ clones were tested using phage ELISAs. Figure 2 shows the top five clones obtained for each affinity selection target. With BAm-green, most new clones showed improved affinity with no loss in specificity (Fig. 2). Among the variants, BAm-green-1.3 proved to have the highest affinity with no loss in selectivity. BAm-red1.0 variants (BAm-red-1.1-1.5) also showed improved affinity (Fig. 2), but similar or reduced specificity for binding to the Pr state over the Pg state, or the Pfr state vs. the Po state (Fig. 2). Affinity matured versions of BAm-red1.0. may be suitable for applications that can tolerate some basal activity. Based on these results BAm-green1.3 and BAm-red1.0 (which also binds the Pfr state) were selected for detailed characterization in vitro.

In vitro characterization of Am1_c0023 binding partners by SEC.

Binder sequences selected from pIII ELISAs were cloned into expression vectors coding for a C-terminal His-tag, expressed in E. coli and purified via Ni-NTA affinity chromatography, 
followed by size-exclusion chromatography (SEC). Based on retention volumes in SEC, all binders were monomeric (Fig. S4). We then used SEC to detect light-dependent interactions between Am1_c0023g2 and BAm variants. When BAm-green-1.3 was mixed with Am1_c0023g2 $\mathrm{PCB}$ in the Pr state, two peaks were observed in the chromatogram with elution volumes matching those observed when Am1_c0023g2 PCB and BAm-green1.3 were injected individually (Fig. 3a). In contrast, when BAm-green1.3 was mixed with Am1_c0023g2 PCB in the Pg state, a new peak eluted with a smaller elution volume. Analysis of elution fractions using SDS-PAGE confirmed the earlier peak was a complex of Am1_c0023g2 PCB Pg and BAmgreen1.3. Exactly opposite behavior was observed with BAm-red1.0 confirming red-light dependent complex formation with Am1_c0023g2 PCB in the Pr state.

(a)

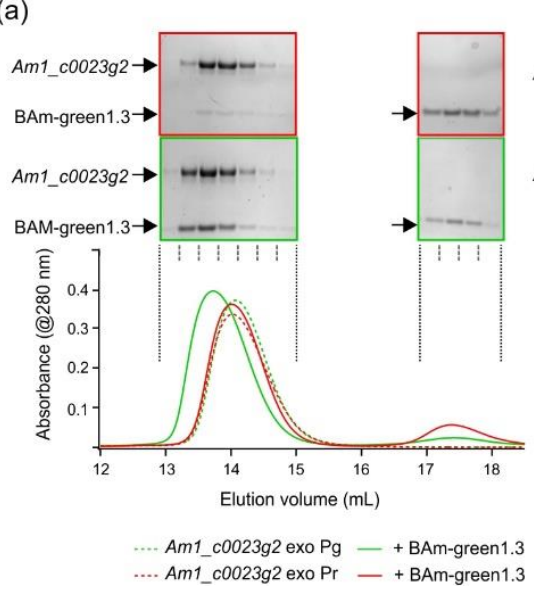

(b)

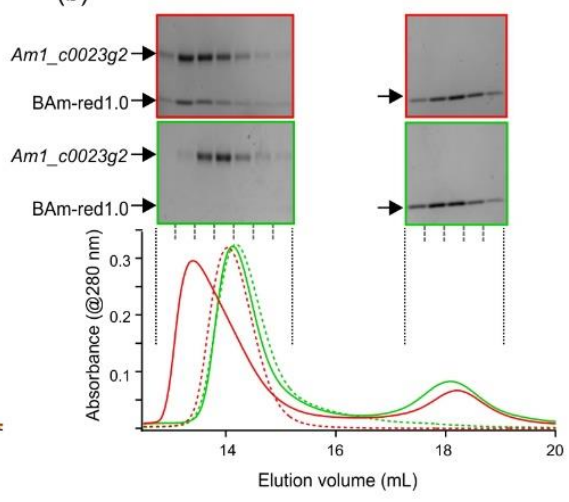

Am1_c0023g2 exo Pg — + BAm-red1.0 ... Am1_c0023g2 exo Pr — + BAm-red1.0

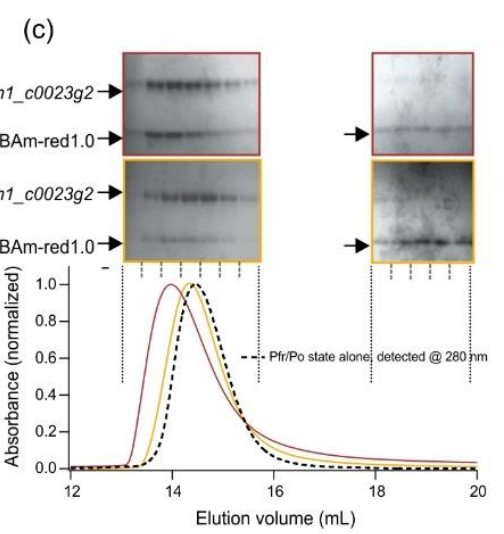

—Am1_c0023g2 exo Pfr + BAm-red1.0 (@600 nm) _ Am1_c0023g2 exo Po + BAm-red1.0 (@700 nm)

Figure 3. Size exclusion chromatography of Am1_c0023g2 forming light-dependent complexes with BAmgreen1.3 or BAm-red1.0. (a) PCB-loaded Am1_c0023g2 exo in the Pg state forms a complex with BAm-green1.3 that elutes earlier than Am1_c0023g2 alone. SDS-PAGE of column fractions shows this complex contains both proteins. Complex formation is not observed with the Pr state. (b) PCB-loaded Am1_c0023g2 exo in the Pr state forms a complex with BAm-red1.0 and does not with the Pg state. (c) BV-loaded Am1_c0023g2 exo in the Pfr state forms a complex with BAm-red1.0. Rapid relaxation from Po to Pfr in the presence of BAm-red1.0 leads to some complex formation when Po is mixed with BAm-red1.0 as detected by SDS-PAGE (and absorbance at $280 \mathrm{~nm}$ (Fig. S5)). Selective detection of the Po state by measuring absorbance at $600 \mathrm{~nm}$, however, indicates very little shift in elution volume in the presence of Bam-red1.0 as compared to Po alone. 
When Am1_c0023g2 BV in the Pfr state was mixed with BAm-red1.0 complex formation was again observed (Fig. 3c). When Am1_c0023g2 BV in the Po state was mixed with BAmred1.0, a significantly smaller shift in elution volume was observed (Fig. 3c). While this result may indicate some affinity of BAm-red1.0 for the Po state, it may also reflect binding to residual Pfr state. As noted above, while thermal relaxation of Am1_c0023g2 PCB from the Pg to the Pr state is very slow $\left(>24 \mathrm{~h}\right.$ at $\left.25^{\circ} \mathrm{C}\right)$, thermal relaxation of $A m 1 \_c 0023 g 2 \mathrm{BV}$ from the Po state to the Pfr state is significantly faster $\left(\tau^{1 / 2}=180 \mathrm{~min}\right.$ at $\left.25^{\circ} \mathrm{C}\right)$. Interestingly, we observed that adding BAm-red1.0 enhanced thermal relaxation in a concentration dependent manner for both $A m 1 \_c 0023 g 2$ PCB and for Am1_c0023g2 BV (Fig. S6). Thermal relaxation, like photoisomerization, of these proteins is likely to involve several intermediate states and associated transition states (44). The observation that BAm-red1.0 catalyzes thermal relaxation implies that it binds the Pg state or an intermediate state and stabilizes a rate-determining transition state involved in the thermal back reaction.

We also tested light-dependent interaction of $A m 1 \_c 0023 g 2$ that had been reconstituted via coexpression of the enzymes required to make either the PCB or BV chromophore (Am1_c0023g2 PCB/BV endo). Size-exclusion chromatography of Am1_c0023g2 PCB endo in the Pg state mixed with BAm-green1.3 showed decreased complex formation compared to that seen with Am1_c0023g2 PCB exo in the Pg state (Fig. S7). This result indicates that BAmgreen 1.3 binding is sensitive to the structural change that underlies the shift in the $\lambda_{\max }$ of the Am1_c0023g2 PCB Pg state observed with exogenously versus endogenously reconstituted protein. In contrast, when Am1_c0023g2 PCB endo in the Pr state was mixed with BAm-red1.0, complex formation was observed by SEC that was indistinguishable from that seen with Am1_c0023g2 PCB exo. 
Affinity and stoichiometry of Am1_c0023 - BAm interactions.

To quantify the affinity of the binder proteins for their targets, we carried out isothermal titration calorimetry (ITC) measurements. Titration of BAm-green1.3 into an ITC cell containing Am1_c0023g2 PCB exo in the Pg state produced the thermogram shown in Figure 4a. These data fit well to a 1:1 binding model with a $\mathrm{K}_{\mathrm{D}}$ of $0.25 \pm 0.1 \mu \mathrm{M}$ and an exothermic heat of binding of $8.2 \pm 0.9 \mathrm{kcal} / \mathrm{mol}$. Titration of BAm-green 1.3 into a cell containing Am1_c0023g2 PCB exo in the Pr state produced the thermogram shown in Figure 4b. As described in the methods section, a fraction of the Pr protein is likely switched to the Pg state during loading of the ITC cell and the thermogram observed is consistent with the presence of $10 \pm 5 \% \mathrm{Pg}$ state. The remaining 90\% Pr state does not produce a measurable heat of binding with BAm-green1.3. The observed lack of a shift in the SEC elution volume (Fig. 3a) indicates the affinity of BAmgreen 1.3 for this state must be $>100 \mu \mathrm{M}$. Thus, BAm-green 1.3 exhibits $>500$-fold selectivity (change in $\mathrm{K}_{\mathrm{D}}$ ) for the Pg state over the Pr state of Am1_c0023g2 PCB exo.

We then measured binding of BAm-red1.0 to Pr-adapted Am1_c0023g2 PCB that had been reconstituted either exogenously or endogenously. The thermogram for BAm-red1.0 binding to Am1_c0023g2 PCB exo is shown in Figure 4b; that for binding to Am1_c0023g2 PCB endo is shown in Figure S8. As expected, based on the SEC results (Fig. 3) both forms bound similarly. In both cases, data fit well to a $1: 1$ binding model with a $\mathrm{K}_{\mathrm{D}}$ of $1.8 \pm 0.5 \mu \mathrm{M}$ (exo) and $0.8 \pm 0.5 \mu \mathrm{M}$ (endo) and with an exothermic heat of binding of $9.5 \pm 0.5 \mathrm{kcal} / \mathrm{mol}$. We found that addition of BAm-red1.0 to Am1_c0023g2 PCB exo in the Pr state caused quenching of Pr-state fluorescence at $\lambda_{\max } \sim 670 \mathrm{~nm}$. This effect was used to independently assess the binding affinity of BAm-red1.0 for the Pr state of Am1_c0023g2 PCB exo (Fig. S9). These data gave a K $\mathrm{D}_{\mathrm{D}}$ of 2.5 $\pm 1 \mu \mathrm{M}$, like that measured by ITC. 

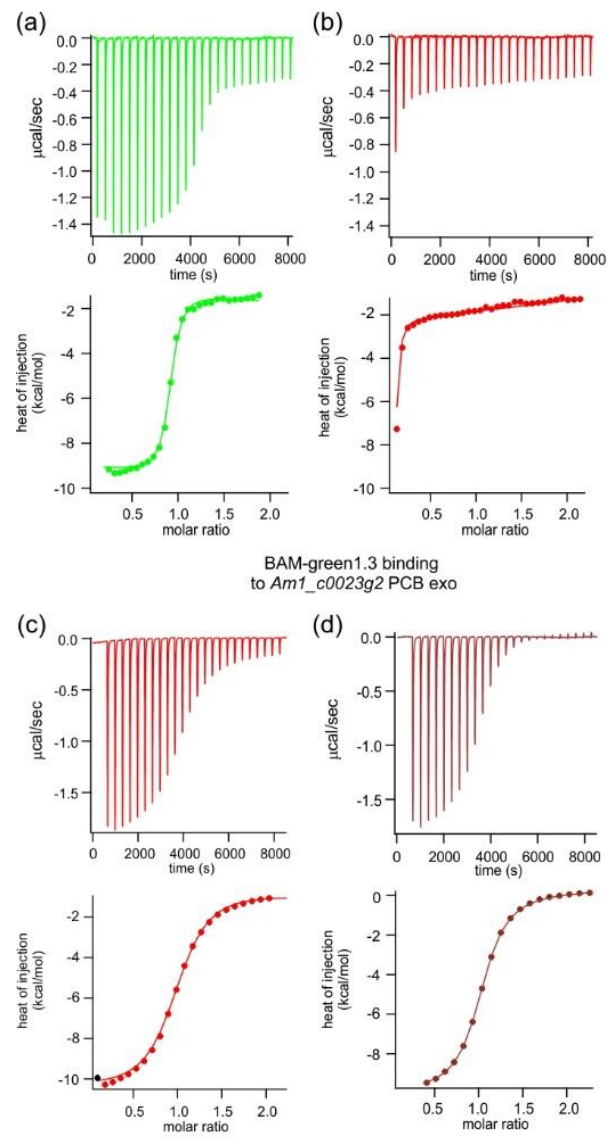

BAM-red1.0 binding to Am1_C0023g2 PCB exo

Figure 4. Isothermal titration calorimetry of BAm-green1.3 and BAm-red1.0 binding to Am1_c0023g2 in a state selective manner. Thermograms are shown in the upper panels and calcuated binding isotherms in the lower panels. (a) BAm-green 1.3 (750 $\mu \mathrm{M}$ in the syringe) was titrated into a solution of Am1_c0023g2 PCB exo $(45 \mu \mathrm{M})$ in the Pg state and thermogram data fitted to a 1:1 binding model to give $\mathrm{K}_{\mathrm{D}}=0.25 \mu \mathrm{M}(0.2-0.4 \mu \mathrm{M})$ and $\Delta \mathrm{H}=-8.2 \pm 0.9$ $\mathrm{kcal} / \mathrm{mol}$. (b) BAm-green1.3 was titrated into a solution of Aml_c0023g2 PCB exo (45 $\mu \mathrm{M})$ in the Pr state and thermogram data fitted to a model in which $10 \%$ of the Pg state remains and the Pr state is inactive. (c) BAm-red1.0 $(550 \mu \mathrm{M}$ in the syringe) was titrated into a solution of Aml_c0023g2 PCB exo $(45 \mu \mathrm{M})$ in the Pr state and thermogram data fitted to a $1: 1$ binding model to give $\mathrm{K}_{\mathrm{D}}=1.8 \mu \mathrm{M}(1.4-1.9 \mu \mathrm{M})$ and $\Delta \mathrm{H}=-10 \pm 0.3 \mathrm{kcal} / \mathrm{mol}$. (d) BAm-red1.0 (550 $\mu \mathrm{M}$ in the syringe) was titrated into a solution of Aml_c0023g2 BV exo (39 $\mu \mathrm{M})$ in the Pfr state and thermogram data fitted to a $1: 1$ binding model to give $\mathrm{K}_{\mathrm{D}}=0.9 \mu \mathrm{M}(0.8-1 \mu \mathrm{M})$ and $\Delta \mathrm{H}=-10 \pm 0.3 \mathrm{kcal} / \mathrm{mol}$.

ITC analysis of BAm-red1.0 binding to the Pg state of Am1_c0023g2 PCB was complicated by the fact that the binder enhances thermal relaxation of Am1_c0023g2 PCB Pg to the Pr state (Fig. S6). The process is slow enough that ITC measurements of BAm-red1.0 binding to Pr state and Pg state samples are qualitatively very different (Fig. S8) but a quantitative measure of the Pg state $\mathrm{K}_{\mathrm{D}}$ by ITC is difficult. The observed lack of a shift in the 
SEC elution volume (Fig. 3b) indicates the affinity of BAm-red1.0 for the Pg state must be >100 $\mu \mathrm{M}$. The observed effect on the thermal relaxation rate (Fig. S6) suggests an affinity of BAmred1.0 for the Pg state, or an intermediate, of $280 \pm 80 \mu \mathrm{M}$. Thus, it appears that BAm-red1.0 exhibits a 200 -fold selectivity (change in $\mathrm{K}_{\mathrm{D}}$ ) for the Pr state over the Pg state of Am1_c0023g2 PCB.

Finally, the binding of BAm-red1.0 to the Pfr state of Am1_c0023g2 BV exo and endo was examined by ITC. These data are shown in Figure 4d and Figure S10. The thermogram data fit well to a $1: 1$ binding model with a $\mathrm{K}_{\mathrm{D}}$ of $0.9 \pm 0.1 \mu \mathrm{M}$ (exo) and $0.75 \pm 0.5 \mu \mathrm{M}$ (endo) with an exothermic heat of binding of $10 \pm 0.5 \mathrm{kcal} / \mathrm{mol}$. Due to rapid thermal reversion of the Po state to the Pfr state, we could not measure a $K_{D}$ for BAm-red1.0 binding to the Po state.

\section{Heterologous expression in living cells as optogenetic tools}

Having established that BAm-green1.3 and BAm-red1.0 showed selective binding to distinct photo-states of the Am1_c0023g2 GAF domain in vitro, we wished to test whether these light dependent binding partners could function in live cells as optogenetic tools. The yeast twohybrid system is a well-established protein-protein interaction validation assay and has been used frequently to test naturally occurring as well as engineered optogenetic tools $(1,45)$. We fused Am1_c0023g2 to either the GAL4 activating domain (GAL4AD) or the GAL4 DNA binding domain (GAL4BD) and the binding partner to the other (Fig. 5a). If the protein partners associate, transcription of a GAL4-regulated lacZ (producing $\beta$-galactosidase) reporter should result. 
Figure 5 shows $\beta$-galactosidase activity measured in yeast cells expressing different combinations of the BAm binders and Am1_c0023g2 in which PCB was added exogenously to the yeast culture. When Am1_c0023g2 was fused to the GAL4 DNA binding domain and BAmgreen1.3 was fused to the GAL4 activating domain, $\beta$-galactosidase activity $40 \%$ of that of the positive control was observed under red light with $<1 \%$ activity under green light $(a>40$-fold difference). When Am1_c0023g2 was fused to the GAL4 activating domain and BAm-green1.3 was fused to the GAL4 DNA binding domain, $\beta$-galactosidase activity $\sim 80 \%$ of that of the positive control was observed under red light with still $<1 \%$ activity under green light $(\mathrm{a}>70$ fold difference).

(a)
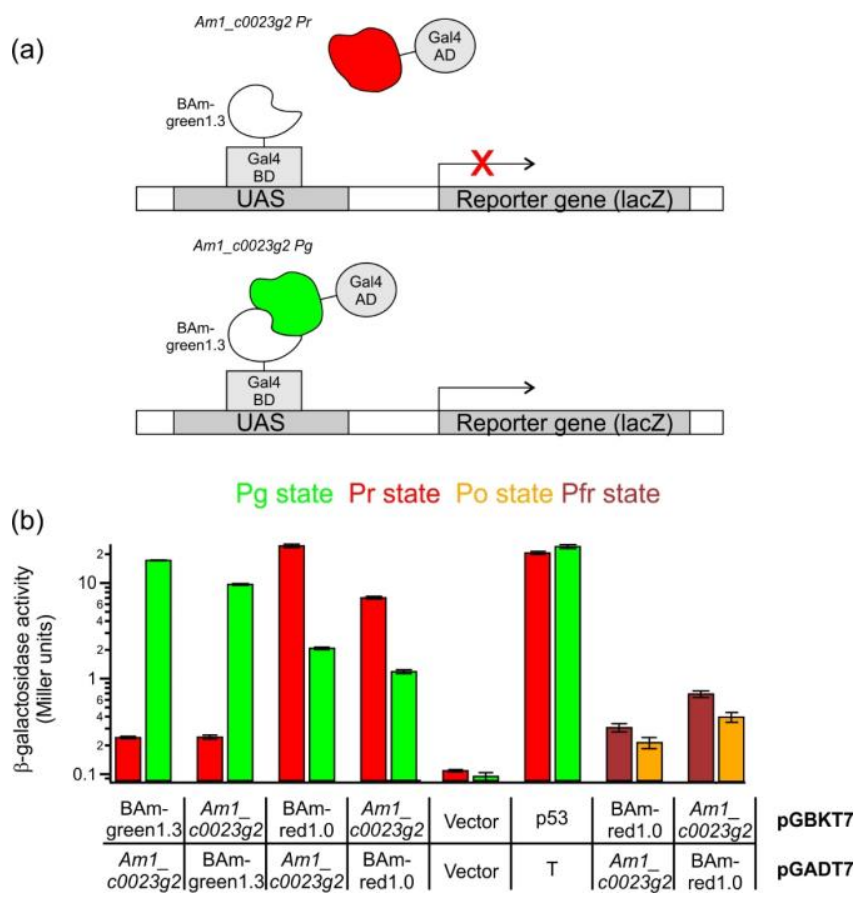

Figure 5. Light induced transcription of a $\beta$-galactosidase reporter in yeast. (a) Schematic of yeast two hybrid with GAL4AD and GAL4BD fusions. (b) Light dependent production of $\beta$-galactosidase activity in yeast (note log scale). Am1_c0023g2 PCB or BV and binder constructs were tested for interaction under different illumination conditions to produce either Pg, Pr (PCB) or Po, Pfr states (see methods). Negative control for no interaction (vector only) and positive control (P53/T) are also shown. 
When the BAm-green1.3 fusions were replaced by BAm-red1.0 fusions the responses to red and green light were reversed as expected. When $A m 1 \_c 0023 g 2$ was fused to the GAL4 DNA binding domain and BAm-red1.0 was fused to the GAL4 activating domain, $\beta$ galactosidase activity $30 \%$ of that of the positive control was observed under green light with < $5 \%$ activity under red light (a 7-fold difference). The fold-difference was improved when the orientation of the binding partners was swapped; i.e. when Am1_c0023g2 was fused to the GAL4 activating domain and BAm-red1.0 was fused to the GAL4 DNA binding domain, $\beta$ galactosidase activity $\sim 100 \%$ of that of the positive control was observed under green light with $<10 \%$ activity under red light (a 12-fold difference). Overall these results are consistent with the in vitro selectivity measured for BAm-green1.3 and BAm-red1.0 for the Pr and Pg states of Am1_c0023g2, i.e. the BAm-green1.3/Am1_c0023g2-Pg state is the most selective interaction. When Am1_c0023g2 was expressed together with BAm-red1.0 in yeast and BV was added to the media, lower apparent degrees of protein-protein interaction in the on-state were observed (Fig. 5b), which may reflect the lower solubility of BV combined with lower uptake vs. PCB by yeast cells (46) or inefficient formation of holo-Am1_c0023g2-BV protein inside yeast. Nevertheless, a reproducible two-fold effect (Pfr vs. Po) on the transcription of $\beta$-galactosidase was observed (Fig. 5b). 


\section{Discussion}

We have described a phage-display selection approach with negative selection against apo-protein and an affinity maturation step that enabled the development of the first binder proteins for a cyanobacteriochrome (CBCR) GAF domain. One-to-one light-dependent binding to the target state was characterized in vitro with affinities between $200 \mathrm{nM}$ and $2 \mu \mathrm{M}$ for the target photo-state and 10- to 500-fold lower affinity for the off-target photo-state. These interactions can be used to enable red light turn-on and green light turn-off of a protein-protein interaction (BAm-green1.3/Am1_c0023g2), or alternatively green light turn-on of a proteinprotein interaction and red-light turn-off (BAm-red1.0/Am1_c0023g2). Whereas BAm-green1.3 binds differentially to $A m 1 \_c 0023 g 2-\mathrm{PCB}$ that has been produced via exogenous addition of PCB versus via co-expression of the enzymes that synthesize PCB, BAm-red1.0 binds to the Pr state whether this has been produced exogenously or endogenously. In both cases, functional responses were demonstrated in vivo in yeast when PCB was added to the growing yeast cells enabling red/green light inducible transcription. We show that neither binder interacts with apo protein, which is likely to be present in any heterologous system.

By replacing PCB with BV, orange and far-red absorbing forms of Am1_c0023g2 were obtained. We found that BAm-red1.0 also binds to the Pfr state of Am1_c0023g2 BV and far-red light produces dissociation of BAm-red1.0/ Am1_c0023g2 BV. Orange light can also be used to trigger the interaction of BAm-red1.0 and Am1_c0023g2 BV. This interaction will also occur via thermal relaxation to the Pfr state in the dark. If delocalized far-red light was used to dissociate BAm-red1.0/Am1_c0023g2 BV, focused orange light could be used to spatially localize a BAmred1.0/Am1_c0023g2 BV interaction. While reconstitution of Am1_c0023g2 BV in yeast was 
inefficient, protein engineering with these systems is already leading to variants with enhanced capability for accepting BV (47), thereby extending their suitability for use in diverse hosts.

Together, BAm-green 1.3 and BAm-red1.0 thus provide new optogenetic components for controlling protein-protein interactions with green, orange, red, and far red light. Because they are based on the GAF domain of a cyanobacteriochrome and a 50-residue GA three-helix bundle protein, they are the smallest green, orange, red and far-red optogenetic tools thus far identified. The approach described here is expected to be suitable for the generation of other cyanobacteriochrome GAF-based optogenetic tools. The rich diversity of the cyanobacteriochrome family offers further possibilities for even more diverse multicolor optical control. 


\section{References:}

1. Hallett RA, Zimmerman SP, Yumerefendi H, Bear JE, \& Kuhlman B (2016) Correlating in Vitro and in Vivo Activities of Light-Inducible Dimers: A Cellular Optogenetics Guide. ACS Synth Biol 5(1):53-64.

2. Goglia AG \& Toettcher JE (2019) A bright future: optogenetics to dissect the spatiotemporal control of cell behavior. Curr Opin Chem Biol 48:106-113.

3. Redchuk TA, Omelina ES, Chernov KG, \& Verkhusha VV (2017) Near-infrared optogenetic pair for protein regulation and spectral multiplexing. Nat Chem Biol 13(6):633-639.

4. Kennedy MJ, et al. (2010) Rapid blue-light-mediated induction of protein interactions in living cells. Nat Methods 7(12):973-975.

5. Toettcher JE, Gong D, Lim WA, \& Weiner OD (2011) Light control of plasma membrane recruitment using the Phy-PIF system. Methods Enzymol 497:409-423.

6. Dagliyan O, et al. (2016) Engineering extrinsic disorder to control protein activity in living cells. Science 354(6318):1441-1444.

7. Guntas G, et al. (2015) Engineering an improved light-induced dimer (iLID) for controlling the localization and activity of signaling proteins. Proc Natl Acad Sci U S A 112(1):112-117.

8. Quejada JR, et al. (2017) Optimized light-inducible transcription in mammalian cells using Flavin Kelchrepeat F-box1/GIGANTEA and CRY2/CIB1. Nucleic Acids Res 45(20):e172.

9. Oliinyk OS, Shemetov AA, Pletnev S, Shcherbakova DM, \& Verkhusha VV (2019) Smallest near-infrared fluorescent protein evolved from cyanobacteriochrome as versatile tag for spectral multiplexing. Nat Commun 10(1):279.

10. Reis JM, et al. (2018) Discovering Selective Binders for Photoswitchable Proteins Using Phage Display. ACS Synth Biol 7(10):2355-2364.

11. Wang H, et al. (2016) LOVTRAP: an optogenetic system for photoinduced protein dissociation. Nat Methods 13(9):755-758.

12. Kolar K, Knobloch C, Stork H, Znidaric M, \& Weber W (2018) OptoBase: A Web Platform for Molecular Optogenetics. ACS Synth Biol 7(7):1825-1828.

13. Ikeuchi M \& Ishizuka T (2008) Cyanobacteriochromes: a new superfamily of tetrapyrrole-binding photoreceptors in cyanobacteria. Photochem Photobiol Sci 7(10):1159-1167.

14. Fushimi K \& Narikawa R (2019) Cyanobacteriochromes: photoreceptors covering the entire UV-to-visible spectrum. Curr Opin Struct Biol 57:39-46.

15. Anders K \& Essen LO (2015) The family of phytochrome-like photoreceptors: diverse, complex and multicolored, but very useful. Curr Opin Struct Biol 35:7-16.

16. Rockwell NC, Martin SS, Feoktistova K, \& Lagarias JC (2011) Diverse two-cysteine photocycles in phytochromes and cyanobacteriochromes. Proc Natl Acad Sci U S A 108(29):11854-11859.

17. Rockwell NC, Martin SS, Gan F, Bryant DA, \& Lagarias JC (2015) NpR3784 is the prototype for a distinctive group of red/green cyanobacteriochromes using alternative Phe residues for photoproduct tuning. Photochem Photobiol Sci 14(2):258-269.

18. Rockwell NC, Martin SS, \& Lagarias JC (2012) Red/green cyanobacteriochromes: sensors of color and power. Biochemistry 51(48):9667-9677.

19. Rockwell NC, Martin SS, \& Lagarias JC (2016) Identification of Cyanobacteriochromes Detecting Far-Red Light. Biochemistry 55(28):3907-3919.

20. Fushimi K, Ikeuchi M, \& Narikawa R (2017) The Expanded Red/Green Cyanobacteriochrome Lineage: An Evolutionary Hot Spot. Photochem Photobiol 93(3):903-906.

21. Narikawa R, Enomoto G, Ni Ni W, Fushimi K, \& Ikeuchi M (2014) A new type of dual-Cys cyanobacteriochrome GAF domain found in cyanobacterium Acaryochloris marina, which has an unusual $\mathrm{red} / \mathrm{blue}$ reversible photoconversion cycle. Biochemistry 53(31):5051-5059.

22. Cornilescu CC, et al. (2014) Dynamic structural changes underpin photoconversion of a blue/green cyanobacteriochrome between its dark and photoactivated states. J Biol Chem 289(5):3055-3065.

23. Lim S, et al. (2014) Photoconversion changes bilin chromophore conjugation and protein secondary structure in the violet/orange cyanobacteriochrome NpF2164g3' [corrected]. Photochem Photobiol Sci 13(6):951-962.

24. Narikawa R, et al. (2013) Structures of cyanobacteriochromes from phototaxis regulators AnPixJ and TePixJ reveal general and specific photoconversion mechanism. Proc Natl Acad Sci U S A 110(3):918-923. 
25. Lim S, et al. (2018) Correlating structural and photochemical heterogeneity in cyanobacteriochrome NpR6012g4. Proc Natl Acad Sci U S A 115(17):4387-4392.

26. Blain-Hartung M, et al. (2018) Cyanobacteriochrome-based photoswitchable adenylyl cyclases (cPACs) for broad spectrum light regulation of cAMP levels in cells. J Biol Chem 293(22):8473-8483.

27. Fushimi K, et al. (2016) Photoconversion and Fluorescence Properties of a Red/Green-Type Cyanobacteriochrome AM1_C0023g2 That Binds Not Only Phycocyanobilin But Also Biliverdin. Front Microbiol 7:588.

28. Kainrath S, Stadler M, Reichhart E, Distel M, \& Janovjak H (2017) Green-Light-Induced Inactivation of Receptor Signaling Using Cobalamin-Binding Domains. Angew Chem Int Ed Engl 56(16):4608-4611.

29. Chatelle C, et al. (2018) A Green-Light-Responsive System for the Control of Transgene Expression in Mammalian and Plant Cells. ACS Synth Biol 7(5):1349-1358.

30. Ong NT \& Tabor JJ (2018) A Miniaturized Escherichia coli Green Light Sensor with High Dynamic Range. Chembiochem 19(12):1255-1258.

31. Chernov KG, Redchuk TA, Omelina ES, \& Verkhusha VV (2017) Near-Infrared Fluorescent Proteins, Biosensors, and Optogenetic Tools Engineered from Phytochromes. Chem Rev 117(9):6423-6446.

32. Shu X, et al. (2009) Mammalian expression of infrared fluorescent proteins engineered from a bacterial phytochrome. Science 324(5928):804-807.

33. Fellouse FA \& Sidhu SS (2007) Making antibodies in bacteria. Making and using antibodies: A practical handbook, eds Howard GC \& Kaser MR (CRC Press, Boca Raton, FL), pp 157-180.

34. Keller S, et al. (2012) High-precision isothermal titration calorimetry with automated peak-shape analysis. Anal Chem 84(11):5066-5073.

35. Brautigam CA, Zhao H, Vargas C, Keller S, \& Schuck P (2016) Integration and global analysis of isothermal titration calorimetry data for studying macromolecular interactions. Nat Protoc 11(5):882-894.

36. Zhao H, Piszczek G, \& Schuck P (2015) SEDPHAT--a platform for global ITC analysis and global multimethod analysis of molecular interactions. Methods 76:137-148.

37. Kelley LA, Mezulis S, Yates CM, Wass MN, \& Sternberg MJ (2015) The Phyre2 web portal for protein modeling, prediction and analysis. Nat Protoc 10(6):845-858.

38. Gambetta GA \& Lagarias JC (2001) Genetic engineering of phytochrome biosynthesis in bacteria. Proc Natl Acad Sci U S A 98(19):10566-10571.

39. Song C, et al. (2015) A Red/Green Cyanobacteriochrome Sustains Its Color Despite a Change in the Bilin Chromophore's Protonation State. Biochemistry 54(38):5839-5848.

40. Xu XL, et al. (2014) Combined mutagenesis and kinetics characterization of the bilin-binding GAF domain of the protein Slr1393 from the Cyanobacterium Synechocystis PCC6803. Chembiochem 15(8):1190-1199.

41. Goglia AG, Wilson MZ, DiGiorno DB, \& Toettcher JE (2017) Optogenetic Control of Ras/Erk Signaling Using the Phy-PIF System. Methods Mol Biol 1636:3-20.

42. Uda Y, et al. (2017) Efficient synthesis of phycocyanobilin in mammalian cells for optogenetic control of cell signaling. Proc Natl Acad Sci U S A 114(45):11962-11967.

43. Fairbrother WJ, et al. (1998) Novel peptides selected to bind vascular endothelial growth factor target the receptor-binding site. Biochemistry 37(51):17754-17764.

44. Kirpich JS, et al. (2019) Reverse Photodynamics of the Noncanonical Red/Green NpR3784 Cyanobacteriochrome from Nostoc punctiforme. Biochemistry 58(18):2307-2317.

45. Pathak GP, Strickland D, Vrana JD, \& Tucker CL (2014) Benchmarking of optical dimerizer systems. ACS Synth Biol 3(11):832-838.

46. Li L \& Lagarias JC (1994) Phytochrome assembly in living cells of the yeast Saccharomyces cerevisiae. Proc Natl Acad Sci U S A 91(26):12535-12539.

47. Fushimi K, et al. (2019) Rational conversion of chromophore selectivity of cyanobacteriochromes to accept mammalian intrinsic biliverdin. Proc Natl Acad Sci U S A 116(17):8301-8309. 